\title{
Chile: ¿autosuficiencia o "autismo" energético? \\ La tensión entre integración regional y sustentabilidad
}

\author{
Antonio Elizalde Hevia* \\ Mario González Gutiérrez **
}

\begin{abstract}
Resumen: El escenario energético de escasez a nivel mundial se empieza a reflejar fuertemente en América Latina. Chile tiene una política energética diseñada en el período dictatorial, y administrada en la democracia, que hace muy vulnerable su economía, su medio ambiente y su seguridad. En este artículo se analizan críticamente los diversos aspectos que inciden fuertemente en el actual escenario energético chileno, sobre todo en lo que respecta a la política de integración con sus vecinos latinoamericanos, desde donde se puede vislumbrar también una solución sustentable a los problemas energéticos futuros.

Palabras clave: energía, integración latinoamericana, sustentabilidad, riesgo energético
\end{abstract}

\section{Chile: autonomy or energetic "autism"?}

\section{The tension between regional integration and sustainability}

Abstract: The worldwide energetic scene of shortage has started to reflect strongly in Latin America. Chile has an energetic policy designed during the dictatorial period and administered in democracy, which turns its economy, environment and safety highly vulnerable. In this article, the diverse aspects that affect strongly in current energetic Chilean scene are analyzed critically, especially those regarding the politics of integration with his Latin-American neighbors, from where it can also be glimpsed a sustainable solution to future energetic problems.

Keywords: Energy, Latin-American integration, sustainability, energetic risk

\footnotetext{
*Universidad Bolivariana. Santiago, Chile. Email: aelizalde@ubolivariana.cl

${ }^{* *}$ Universidad Bolivariana. Santiago, Chile. Email: mgonzalez@ubolivariana.cl
} 


\section{Introducción}

Este artículo busca explicar cómo el tema energético se constituye en una limitante muy seria para la sustentabilidad del modelo de acumulación existente en Chile, ya que el crecimiento de la economía está basado principalmente en exportaciones de recursos naturales requirentes de energía barata para continuar siendo competitivos a nivel internacional.

Otro objetivo de este artículo es el de explicar el alejamiento de Chile de los procesos de integración regional, con lo cual se ha generado una preocupación mayoritaria en la población porque la inserción en esos espacios pueda traducirse en una eventual pérdida de avances logrados en términos de calidad de vida y de estabilidad democrática, ante la volatilidad de los acuerdos suscritos.

La actual matriz energética del país es notablemente precaria ya que es muy dependiente del gas natural de los países vecinos, lo cual obliga a Chile a desarrollar una política de buen vecino y viabiliza una mayor integración con éstos. Pero ésta a su vez puede incrementar los riesgos de contaminación de los climas políticos internos de esos países caracterizados por una relativa inestabilidad.

La política energética se constituye así en un elemento clave para avanzar hacia mayor integración con nuestros vecinos o hacia una mayor dependencia de los mercados globales, pero también hacia una política que permita al país avanzar hacia una sociedad más sustentable. Se analizan en consecuencia las distintas alternativas a las cuales Chile se enfrenta para resolver su problema energético.

\section{El "modelo" chileno}

La sociedad chilena es casi un caso de texto. Su política económica ha seguido desde sus inicios la evolución del pensamiento económico con una capacidad notable para asumir acríticamente y llevar a cabo en forma rigurosa las recetas que sugieren las escuelas del pensamiento económico. Es así como en el siglo XIX se aplicaron indiscriminadamente las políticas económicas aperturistas recomendadas por el pensamiento librecambista. ${ }^{1}$

La crisis de los años 30 golpeó a la economía chilena en un grado mucho mayor que al resto de las economías latinoamericanas debido a su mayor exposición, cuestión que nos llevó a adoptar tempranamente el modelo de desarrollo "hacia adentro" y sustitutivo de exportaciones con un fuerte rol dinamizador por parte del Estado. La pequeñez de los

\footnotetext{
${ }^{1}$ Ver al respecto el excelente trabajo de Aníbal Pinto Chile, un caso de desarrollo frustrado, Editorial Universitaria, Santiago, 1959. 
mercados internos llevó al pronto agotamiento del llamado modelo de industrialización por sustitución de importaciones, una vez superada su etapa "fácil". De modo que a fines de los sesenta o comienzos de los setenta este modelo se encontraba prácticamente agotado, con un crónico desequilibrio inflacionario y lentitud del crecimiento económico, pese al importante incremento del tamaño del mercado interno, gracias a las reformas estructurales realizadas durante los gobiernos de Frei y Allende. Es importante señalar aquí un dato de no menor importancia para sustentar la afirmación anterior, cual es que recién a partir del año 1967 el campesinado chileno comenzó a percibir sus remuneraciones en metálico y ellos constituían en esa fecha alrededor del 30 por ciento de la población total.

Durante el gobierno militar, a partir de mediados de los años setenta, se implanta en forma progresiva el modelo neoliberal que impulsa una apertura a ultranza de nuestra economía. La radicalidad de dicha implantación conduce a la quiebra y cierre de parte importante de la industria sustitutiva de exportaciones que se había desarrollado en el país durante las últimas cuatro décadas. Retornaba así el país al antiguo modelo monoexportador de materias primas, principalmente de origen minero y en particular de cobre, aunque iniciando una relativa diversificación de sus exportaciones a partir del desarrollo de una incipiente industria exportadora de productos silvo-agrícolas basada en la explotación de la amplia superficie de plantaciones de pino insigne y eucaliptos realizadas a partir de fines de los cincuenta (madera, papel y celulosa), además de productos pesqueros (harina de pescado) y productos agrícolas (frutas y vino). De modo tal que el modelo aperturista, sumado al período recesivo derivado de la crisis de los petrodólares a comienzos de los ochenta, terminaron golpeando aún más fuertemente al sector industrial, y en especial a las medianas y pequeñas empresas, generando una situación de crisis generalizada que afectó incluso al sistema bancario y que condujo a niveles de desempleo abierto sólo similares a los experimentados por el país en los años 30 (superiores al 25 por ciento de la población económicamente activa). El sistema bancario tuvo que ser auxiliado desde el Estado para evitar su desplome a un costo superior a los siete mil millones de dólares (subsidios bajo la forma de créditos blandísimos y a tasas bastante inferiores a las de mercado).

\section{La metamorfosis económica y social de Chile}

El ensayo neo-liberal que vivió Chile durante la década del 70 y del 80 es una confirmación más de que lo que se intentó hacer, con o sin éxito según el autor que se consulte, fue imponer desde el Estado un nuevo modelo económico-político. La tradición histórica, no sólo en términos de su característica democrática, sino también en el sentido de un tipo de modernización "gradualista", fue totalmente cambiada por un tipo de Estado que imponía un modelo muy cercano a las tendencias que tomaba la modernización a escala internacional. Es en este sentido que los sectores más desposeídos se ven notoriamente afectados. El modelo es impuesto desde arriba y los sectores populares deben incorporarse a éste "como mejor puedan". 
Es posible afirmar, casi sin lugar a dudas, que el modelo neoliberal no habría sido posible de implantar sin el auxilio de un régimen dictatorial como el que Chile vivió durante casi dos décadas. La destrucción sistemática del movimiento sindical, el desmantelamiento de los poderes parlamentario y judicial, la disolución de los partidos políticos, el control de los medios de comunicación y la represión permanente de las organizaciones políticas progresistas, generaron condiciones que inhabilitaron la posibilidad de respuesta popular al desmantelamiento que se llevó a cabo del estado de bienestar que se había alcanzado, el cual conculcó los derechos sociales y económicos logrados por las luchas sociales de casi un siglo, perdiéndose así gran parte de los avances que históricamente habían conquistado las clases asalariadas.

Es innegable que los cambios que provocó la intervención militar afectaron todas las esferas de la sociedad chilena. En lo que dice relación con la economía del país, fueron muy profundas las transformaciones que se llevaron a cabo para implementar un nuevo modelo.

El régimen que nació del golpe de estado de 1973 actuó "drásticamente para desmantelar el modelo de desarrollo precedente, lo que se tradujo en el estancamiento o reversión de muchos de los procesos modernizadores anteriores (des-industrialización, redistribución negativa del ingreso, caída del empleo, y de los salarios, reducción del gasto social del Estado, etc.)". (Tironi, 1990:22)

El gobierno militar intentó proyectar una imagen de seguridad y prosperidad. El nuevo modelo de desarrollo era la solución a todos los problemas que había sufrido nuestro país. Pero frente a esta imagen, estaba la verdadera realidad, la que todos los días vivían los chilenos.

"El intento antidemocrático de modernización, ha tenido un gran costo humano. También ha debilitado la estructura económica haciendo muy difícil dar un salto adelante. La división interna, la exclusión, la desigualdad y el rezago productivo constituyen cuatro grandes obstáculos que no pueden superarse con el esquema político y económico actual". (Bitar, 1988:55)

Estos cambios redujeron el peso numérico de los conglomerados más característicos del tipo de desarrollo previo a 1973. La burocracia del aparato del Estado se contrajo notablemente durante este período, entre 1973 y 1978, 100.000 funcionarios del sector público fueron expulsados de sus puestos de trabajo. La clase obrera se redujo en más de la mitad, entre el período 1971-1982 y el asalariado agrícola lo hizo en casi un cuarenta por ciento. Por otra parte los sectores no-asalariados y "marginales" aumentaron en forma desproporcionada. En el mismo período se triplicaron los cesantes, los desocupados y los que buscan empleo por primera vez.

Mientras en la mayoría de las naciones del mundo durante el mismo período las condiciones de vida de gran parte de los habitantes mejoraron, en década y media de 
REVISTA POLIS

VOL. $7 \mathrm{~N}^{\circ} 21,2008$

PRÒLOGO, págs., 37-62

( ) EDITORIAL DE LA UNIVERSIDAD BOLIVARIANA DE CHILE, 2008

gobierno militar en Chile se deterioró sustancialmente. En 1988 el consumo per cápita era inferior al de 1970. El Producto Geográfico Bruto (PGB) por persona en 1987 era inferior al de 1971. El producto por persona, entre 1981 y 1987, disminuyó en más de un 6\%. El gobierno de Pinochet no hizo sino deteriorar la calidad de vida de la mayoría de la población. Los salarios reales entre 1981 y 1987 cayeron cerca de un 13\%, a la vez que el salario mínimo se redujo en un 33\% durante el mismo período. Estas cifras son elocuentes a la hora de contrastar los logros alcanzados, en mantener equilibrios macroeconómicos o superávit en la balanza comercial, con el gran fracaso de permitir que los sectores más postergados de la sociedad pudieran tener acceso a oportunidades para salir de la pobreza.

"Estamos, pues, en presencia de un período de marginalización extendida, en el cual la pobreza urbana es creciente y segregada espacialmente (aunque el territorio segregado es casi mayor que el "integrado"), y donde lo que define la vida laboral de esos sectores es cada vez más la inestabilidad. Al mismo tiempo, su calidad de vida como conjunto se deteriora y se bloquean los mecanismos históricos de movilidad social". (Campero, 1978:35-36)

En el sector industrial, las ramas metalmecánica y textil que habían sido las más desarrolladas entre 1938 y 1973, dejaron de ser el eje de dicho sector económico, y si bien no desaparecieron del todo, sí se contrajeron notablemente. El desarrollo industrial se centró en actividades relacionadas con el sector exportador. Chile quería incorporarse a los mercados internacionales a cualquier costo. Esto significó también que los trabajadores en su calificación tuvieran que adecuarse a las demandas de estos mercados y que a la vez los salarios y las fluctuaciones en el mercado de trabajo dependieran de los acontecimientos internacionales.

Los costos pagados por la sociedad chilena en su inserción a los mercados globales fueron de una magnitud tal, que dicho proceso no habría sido posible sin un contexto político autoritario de absoluta restricción al ejercicio de los derechos humanos de primera, segunda y tercera generación.

Sin embargo, es importante considerar que desde fines de los ochenta, y más aún a partir del retorno a la democracia, la economía chilena comenzó a experimentar un acelerado proceso de crecimiento a tasas excepcionalmente altas para lo acostumbrado históricamente, lo cual llevó a más que duplicar en una década el producto per cápita. Esto sumado al propio proceso de recuperación democrática y a los nuevos énfasis en las políticas públicas, se tradujo en una significativa reducción de los niveles de población en situación de pobreza con que había culminado el período dictatorial, bajando desde alrededor de un 40 por ciento de la población total a menos de un 20 por ciento.

“El porcentaje de la población bajo el nivel de pobreza descendió del 38,6\% en 1990 
al $18,8 \%$ en 2003 y el de indigencia descendió del 12,9\% en 1990 al 4,7\% en 2003." (Castells, 2005:66)

Desde una mirada anclada en la consideración de las dimensiones ambientales, Rayén Quiroga afirmó, hace más de una década atrás, que:

"El proceso de apertura y desregulación de la economía chilena generó expansiones significativas en el nivel de actividad económica agregada, lo que provocó un incremento del transflujo de magnitud suficiente para implicar un peligroso acercamiento al punto de resiliencia de los ecosistemas chilenos. Por esto, la expansión infinita de nuestras exportaciones, como el crecimiento económico ilimitado, son imposibles... La conclusión más importante que se deriva de la investigación realizada es que el actual camino de crecimiento económico chileno no es capaz de sostenerse en el futuro. Todo tigre, por más emprendedor que sea, necesita de la selva que le sustenta... Y esto es exactamente lo que nos está pasando, nos estamos convirtiendo en un supuesto tigre, voraz, gallardo, que se está quedando sin selva." (Quiroga, 1994:473)

Marcel Claude sostiene a su vez la tesis de que:

“... un factor consustancial a los procesos de creación de ingreso en Chile, desde la época de la independencia hasta nuestros días, lo constituye la extracción y exportación de recursos naturales. Esto es así, independientemente de los distintos y contrapuestos modelos de desarrollo que han predominado en los diferentes tiempos de nuestra historia. No sería novedad, entonces, la verificación -una vez más- de condiciones estructurales que no aseguran la sustentación de los procesos de generación de ingreso y en consecuencia, de la existencia de dinámicas y características que sumen a este país cada cierto tiempo en períodos de auge y riqueza y períodos de declinación y miseria." (Claude, 1997:24)

Señala asimismo que:

"Lo novedoso, hoy en día, lo constituye no sólo la creciente importancia de la "cuestión ambiental" y de las exigencias de sustentabilidad para los modelos de desarrollo, sino también la autoafirmación por parte de las dirigencias empresariales y políticas de que el actual proceso de desarrollo vigente en Chile asegura su propia sustentabilidad. La mayoría de los dirigentes empresariales vinculados a la extracción de recursos naturales, como las pesquerías y los bosques, insistentemente aseguran, a través de los medios de comunicación, la existencia de condiciones de sustentabilidad en sus respectivos rubros productivos." (ibidem)

En un trabajo posterior de la misma Rayén Quiroga con Saar Van Haurwermeiren (1996), ambas afirman que:

"Los resultados macroeconómicos del "milagro chileno" al mismo tiempo provocaron y se sustentaron en significativos deterioros tanto sociales como ambientales. En esencia, 
los trabajadores, el medio ambiente (recursos naturales incluidos) y las generaciones futuras subsidiaron y subsidian la nueva economía chilena. Lo grave de esta situación es que el deterioro ambiental y distributivo se han transformado en ventajas absolutas para la competencia en el escenario económico internacional, comprometiendo desde el inicio la potencialidad desarrollista del proceso económico chileno, y dificultando seriamente la sustentabilidad del mismo en el mediano y largo plazo.” (1996: 186)

\section{La situación de la energía en Chile: un desafío para la sustentabilidad del modelo}

Según Pedro Maldonado y Gonzalo Castillo, autores del estudio sobre situación de la energía del Programa Chile Sustentable:

"El sector energético en Chile enfrenta desde hace más de dos décadas problemas tales como la dependencia de combustibles fósiles importados; la inseguridad y vulnerabilidad en el suministro; los impactos ambientales y sociales de los proyectos energéticos; el encarecimiento de los servicios y consecuentemente los problemas de equidad en el acceso; de cobertura en las zonas lejanas a las redes eléctricas; y la falta de eficiencia, traducida en un incremento sostenido de la demanda energética, que supera el crecimiento económico." (Chile Sustentable, 2004:5)

En el mismo informe de Chile Sustentable, se señala que dicha situación se ha agravado por un contexto internacional de creciente vulnerabilidad, insustentabilidad e inseguridad. Ello debido principalmente al fuerte uso de energías convencionales (combustibles fósiles y megaproyectos) y la falta de eficiencia en su aprovechamiento, a nivel de países y regiones.

Si a esto sumamos los problemas ambientales derivados de la quema de combustibles fósiles -como el efecto invernadero y el cambio climático-, los problemas políticos y económicos derivados del progresivo agotamiento de dichas fuentes, la creciente demanda energética y los impactos de los grandes emprendimientos energéticos en el medio ambiente y las comunidades (como ocurre con las mega represas hidroeléctricas), se hace evidente la centralidad del desafío de implementar políticas energéticas sustentables en nuestras sociedades.

Ello nos exige modificar sustancialmente la matriz energética, con el objeto de reducir la independencia, la vulnerabilidad y la inseguridad. Junto a esto, mejorar la equidad y revertir los daños ambientales. En atención a ello debemos reducir progresivamente el uso de fuentes convencionales y fósiles, e incrementar en forma sostenida el aprovechamiento de las energías renovables no convencionales, tales como la geotermia, biomasa, pequeñas hídricas, eólica, solar, mareomotriz, y aumentar la eficiencia energética. 
Desde la mirada de Chile Sustentable, se sostiene que las tendencias actuales no parecen responder a tales objetivos, pese a la urgencia de este desafío. Esta urgencia está expresada en las previsiones realizadas por organismos internacionales especializados, quienes indican que hacia el año 2020, persistirá a nivel mundial una fuerte dependencia de los combustibles fósiles, llegando a representar un $73,5 \%$ de las fuentes utilizadas para generación eléctrica.

Chile no constituye una excepción a esta tendencia pues la orientación en la toma de decisiones del sector es la reducción de exigencias para la inversión de los agentes privados, en tanto los impactos de corto, mediano y largo plazo que implica el mantener una matriz energética no sustentable, son apenas considerados. Paralelo a esto, el patrón de producción y consumo vigente en nuestro país podría agudizar la tensión entre una cada vez más encarecida oferta y una creciente e insatisfecha demanda.

A lo anterior se agrega que Chile se abastece principalmente de combustibles fósiles importados y megaproyectos energéticos (centrales hidroeléctricas de gran escala), lo que se traduce en graves problemas de vulnerabilidad y dependencia. La mayor parte de los nuevos emprendimientos energéticos, según consta en el más reciente Plan de Obras de la Comisión Nacional de Energía (2003) continúa utilizando estas fuentes. Los informes señalan que casi el $70 \%$ del consumo energético primario y secundario en el país se satisface con combustibles fósiles, de los cuales más de la mitad corresponden a petróleo y a gas natural importados, y no se han adoptado medidas para revertir o evitar los negativos impactos ambientales que genera el uso de estos combustibles.

$\mathrm{Si}$ atendemos por otra parte al consumo energético, mientras que Estados Unidos alcanza casi 8 tep per cápita ${ }^{2}$ y los países de la OECD consumen 4.68 tep per cápita, nuestro país alcanza entre 1,88 y 1,07 tep por habitante al año. ${ }^{3}$ Si bien esta cifra está lejos de los niveles de los países del Norte, casi duplica el consumo de energía de la región latinoamericana.

En síntesis, las políticas públicas orientadas a resolver las necesidades fundamentales de la población y mejorar la productividad de las actividades económicas en Chile, ciertamente se traducirán en aumentos del consumo de energía, provocando impactos que afectarán negativamente la sustentabilidad del desarrollo del país; salvo que se definan drásticas políticas para revertir el actual curso del desarrollo energético nacional, en el marco de la sustentabilidad política, social, económica y ambiental.

Por tanto es necesario postular que la política energética en Chile no ha abordado en su integridad ni ha asignado la importancia que corresponde al desarrollo sustentable con equidad. Persisten los problemas de cobertura en las zonas aisladas y de acceso en los

\footnotetext{
${ }^{2}$ Tep: Toneladas de petróleo equivalente

${ }^{3}$ Los valores de consumo de energía per cápita corresponden al año 2001 y provienen de Key World Energy Statistics, 2003, de IEA. 
sectores más pobres, lo que se evidencia por ejemplo en el fuerte consumo de leña en familias de escasos ingresos, que recurren a este combustible debido a la falta de recursos para acceder a otras fuentes de energía más limpias.

Finalmente es importante destacar que la actual matriz energética de Chile es una de las tantas herencias de la dictadura, ya que la privatización y transnacionalización del sector energético en el país (especialmente en el ámbito de la electricidad) ha favorecido la instalación de un régimen de mercado energético, que al basarse exclusivamente en los criterios de la oferta y la demanda, privilegia la venta de energía como mercancía, por encima de la satisfacción de las reales necesidades energéticas en la población, causando que las tarifas energéticas se alejen de la capacidad de pago de amplios sectores.

\section{La energía: ¿cuello de botella para la expansión del modelo o instrumento para una mayor integración en el espacio regional?}

Según el Programa Chile Sustentable, la actual matriz energética del país es de una notable dependencia externa.

"Vemos que Chile importa un $99 \%$ de sus necesidades de petróleo, un $82 \%$ de sus necesidades de carbón, un $71 \%$ de sus necesidades de gas natural, con respecto al consumo bruto de energía primaria. ${ }^{4}$ Dadas estas cifras, la importación de combustibles fósiles tiene un significativo impacto sobre la balanza de pagos del país. Más aún, nuestro abastecimiento energético de gas natural depende, en su totalidad, de yacimientos argentinos. En el caso de la zona central, dependemos de dos gaseoductos provenientes de la zona de Neuquén, pero la cuenca de Neuquén, es la cuenca gasífera con más años de explotación en Argentina y sus reservas son muy escasas, no superando los 12 a 14 años de vida útil. Aún cuando existieran nuevas reservas, los precios del gas natural probablemente podrían subir en forma apreciable, debido a que se encuentran íntimamente ligados a los precios de los hidrocarburos y al desarrollo tecnológico." (Chile Sustentable, 2005:109110)

Un reportaje de la Revista del Campo de El Mercurio señalaba hace algunos años atrás que según previsiones de especialistas, para 2010 Chile estaría $10^{\circ}$ en el mundo en exportaciones agroalimentarias, ello en razón de que es el país con la mayor tasa de crecimiento del sector en la última década, un $132 \%$.

"Para 2010, Chile estaría en los top ten de los exportadores de alimentos con envíos, según varíe el optimismo, que irían entre los US\$14 mil millones y los US\$ 17 mil millones."

${ }^{4}$ Balance de la Comisión Nacional de Energía (CNE), 2002. 
Sin embargo, en el mismo artículo se señala que:

"Para dar la talla de potencia agroalimentaria, Chile requiere en los próximos quince años aumentar su potencial energético en 10 mil megas e inversiones por US\$ 13 mil millones para generarlos... Aunque el país tiene condiciones geográficas para abastecerse de varias formas, plantas hidroeléctricas, a carbón y nucleares, entre otras, la pasada crisis del gas argentino ratifica la feble postura chilena..., el tema pasa por poner tarifas que paguen e incentiven nuevas inversiones... La autonomía energética, en tal sentido, es un tema clave para llegar a ser potencia agroalimentaria." 5

Las alternativas que se abren para resolver la principal limitante para la continuidad del modelo de crecimiento son varias. Examinaremos a continuación cada una de ellas y sopesaremos algunas de sus ventajas y desventajas.

\section{El acceso al gas natural cercano: el abastecimiento desde Bolivia y/o Argentina}

La primera alternativa se basa en acceder al abastecimiento de gas natural, cuyos proveedores son justamente los países vecinos: Bolivia y Argentina. En el caso de Argentina con quien se habían suscrito acuerdos comerciales que llevaron al desarrollo de toda la infraestructura requerida para su transporte (gasoductos entre ambos países) y la instalación de varias centrales de ciclo combinado, la reducción de las cuotas acordadas debido a problemas de abastecimiento interno por parte de Argentina derivada de la falta de inversiones en el sector, generó una situación de desabastecimiento y a su vez produjo una pequeña crisis en algunos sectores productivos.

Es difícil pensar que el actual gobierno argentino eleve los precios de la energía para atraer inversiones al sector, de modo tal que los acuerdos comerciales suscritos quedarán en los hechos en letra muerta.

En el caso de Bolivia, la situación es más compleja, ya que los sectores nacionalistas, ampliamente mayoritarios en el país, se oponen abiertamente al abastecimiento con gas natural boliviano de la economía chilena. Incluso se produjeron importantes movilizaciones y conflictos internos, en razón de la eventual salida por la costa chilena del gas natural boliviano destinado a mercados del primer mundo. Es importante señalar aquí que para enfrentar su crisis energética interna, Argentina debió recurrir al abastecimiento con gas natural boliviano, y que el gobierno de este último país prohibió expresamente que este fuese desviado directa o indirectamente hacia Chile. La diplomacia externa boliviana, cuyo principal objetivo es el acceso directo y autónomo al mar, considera su gas natural como un instrumento muy poderoso para una eventual negociación con Chile en tal sentido.

\footnotetext{
${ }^{5}$ ¿El nuevo sueldo de Chile? Revista del Campo, Año XXX, № 1539, 9 de enero de 2006
} 
Es importante tener en cuenta que la problemática principal del gas natural radica en su costo elevado de transporte. Es así como, para ciertas grandes distancias y particularmente donde el volumen no es el adecuado, se vuelve más un negocio de transporte que de gas natural. Lo que complica el escenario regional es que Bolivia, primero a través de una Ley de Hidrocarburos que no acompaña futura inversión y además con los disturbios y el caos social que se vivió hace algunos meses, con la toma de campos petroleros, cierre de válvulas y demandas de nacionalización, puede estar enviando señales de que no es un país serio para ser considerado como fuente continua y segura de energía. Esta señal de incertidumbre y de país inviable contrasta sin embargo con la historia. Durante 26 años Bolivia ha exportado gas natural a la Argentina sin interrupción alguna, exporta continuamente gas natural a Brasil desde 1999, y se exportan hidrocarburos líquidos por mas de 30 años a través del oleoducto que va hasta Arica. Nunca ha habido interrupciones.

\section{La búsqueda de energía en el mapa global: el gas indonesio}

La cuasi crisis económica y política derivada de la restricción puesta desde Argentina al abastecimiento de gas natural gatilló una casi frenética búsqueda por parte del gobierno chileno de otras fuentes de abastecimiento, fuera del entorno regional. Es así como -según lo informó la prensa en su momento- se firmó con Indonesia un acuerdo preliminar para suministrar entre 2 y 4 millones de toneladas anuales de gas natural licuado a Chile. El ministro de Energía, Purnomo Yusgiantoro, señaló a los medios de comunicación que, tras la firma de un memorando de entendimiento, los representantes de BP Migas -el organismo regulador que tiene el gobierno de Indonesia para el sector de petróleo y gas- viajaría a Chile para discutir los detalles, incluidos los precios. Según la misma fuente, Chile quiere el gas natural licuado a partir del 2007 o fines del 2008 y a su vez Indonesia tiene suficientes reservas de gas como para satisfacer esa demanda. Yusgiantoro también señaló que primero le preguntaría al gigante petrolero británico British Petroleum si podría construir una tercera planta dentro de su proyecto de gas natural licuado en la provincia de Papúa, para abastecer a Chile.

\section{El cambio hacia una matriz energética nuclear y el riesgo asociado a ello}

Hay voces aisladas que desde hace ya algún tiempo están planteando en Chile la opción por la energía nuclear, puesto que consideran que el país tiene la capacidad tecnológica y financiera como para poder optar por esta solución; sin embargo cuando se

\footnotetext{
${ }^{6}$ La información presentada en este acápite proviene del artículo de Tom Azzopardi, Energía Nuclear: ¿Es Viable en Chile?, Business Chile, Diciembre 2005, N²30 en http://www.businesschile.cl/portada.php?w=old\&id=188\&lan=es
} 
produjo la restricción unilateral en el suministro de gas natural proveniente de Argentina, la alternativa de la energía nuclear, alguna vez un remoto proyecto, ya no pareció ser tan improbable.

"Si queremos tener energía limpia y segura para el futuro... debemos pensar en la energía nuclear; sí, energía nuclear". Estas palabras corresponden al Presidente Ricardo Lagos cuando en su mensaje acerca del estado de la Nación el 21 de Mayo de 2005, habló respecto de la necesidad que tiene Chile de asegurar su suministro de energía frente a una escasez de gas natural y al incremento pronunciado en los precios mundiales de los combustibles.

Sus palabras coinciden con el renovado interés internacional en la energía nuclear. En Estados Unidos, donde no se ha construido ninguna planta de energía nuclear desde los años setenta, el Presidente George W. Bush firmó hace ya un tiempo una ley sobre energía que incluye incentivos para nuevas inversiones en la industria. Y en Finlandia ya se han iniciado los trabajos en lo que será la primera planta de energía nuclear en Europa en una década, mientras que en otros países, tales como el Reino Unido, ya se está discutiendo la necesidad de reemplazar sus anticuados reactores. Hay tres factores que explican este interés renovado: el incremento de los precios de la energía, el desarrollo de nuevas técnicas y una creciente preocupación acerca del impacto ambiental de los combustibles fósiles.

Según la World Nuclear Association (WNA), la energía nuclear en la actualidad es competitiva con los combustibles fósiles, excepto en aquellos países donde estos combustibles están directamente a disposición de los consumidores a un bajo precio. Chile queda excluido de esta condición ya que virtualmente no posee reservas de gas, petróleo o carbón.

Con los precios del petróleo que han alcanzado niveles récord y aquellos de otros combustibles fósiles que también se están incrementando, la energía nuclear está pasando a ser más competitiva en costos. El principal combustible de la tecnología, el uranio, tiene actualmente una abundante disponibilidad y se comercia a precios extremadamente bajos; y los desarrollos tecnológicos significan que la energía nuclear no sólo es más competitiva que hace una década atrás, sino, también, mucho más segura.

El otro factor que está a favor de la energía nuclear, es la creciente preocupación acerca de los así denominados gases del «efecto invernadero». Con la implementación del Protocolo de Kyoto, los gobiernos y empresarios están más conscientes que nunca de la necesidad de reducir las emisiones de dióxido de carbono y de otros gases que están conduciendo a un calentamiento de la atmósfera terrestre.

A diferencia de las fuentes de energía convencional que se apoyan en la utilización de combustibles fósiles, la energía nuclear prácticamente no produce ningún tipo de emisiones de tipo efecto invernadero. Como resultado de ello, los gobiernos de Europa y de EE.UU. la 
han identificado -en conjunto con otras fuentes renovables y para un uso más eficiente de la energía- como un posible medio de eliminar las emisiones en el futuro. Ello ha llevado a que se sostenga que esta forma de energía puede ser un eficiente medio para combatir el inminente desastre que sería ocasionado por el gran crecimiento de los niveles del mar.

Aunque los estándares de seguridad de la industria de energía nuclear han mejorado significativamente desde el desastre de Chernobyl, de 1986, una falla de un reactor nuclear puede ser, por supuesto, bastante más devastador que un accidente ocasionado en un generador convencional; y en Chile también se debe considerar que existe un nivel de riesgo sísmico permanente. Más aún, con posterioridad a los ataques terroristas en EE.UU. y Europa, el riesgo de un ataque a una planta nuclear, o el robo de materiales radioactivos, constituyen una preocupación importante para los sectores antinucleares.

De un modo similar, también existe el serio problema acerca de cómo almacenar los materiales de desecho que pueden permanecer radioactivos por cientos de años. La industria nuclear aún no ha proporcionado una respuesta a esta interrogante, o acerca de cómo desmantelar los reactores en forma segura, una vez que su vida útil ha terminado. Por lo cual es posible pensar que si se incluyen los costos de desmantelamiento y de almacenamiento, los precios se eleven y la energía nuclear deje de ser competitiva. Existe la tecnología para almacenar materiales radiactivos en forma segura y ya algunos países se están preparando para el costo a largo plazo, mediante la asignación de una parte de los ingresos actuales provenientes de la venta de energía. Los costos de desmantelamiento estimados por la WNA son equivalentes al $9 \%$ ó $15 \%$ del costo inicial de capital de una planta de energía nuclear.

Es importante además, tener presente que las primeras plantas comerciales de energía nuclear entraron en operación hace medio siglo atrás y en la actualidad existen alrededor de 440 reactores situados en 31 países, los cuales producen cerca del 16\% de la energía eléctrica del mundo; mientras que las plantas de combustión a carbón proporcionan el 39\%, delante de las plantas hidroeléctricas (19\%), las plantas de combustión a gas (15\%) y las plantas de combustión a petróleo $(10 \%)$.

Por otra parte en América Latina la energía nuclear no es nueva. Argentina posee dos reactores en operación con una capacidad combinada de casi $1.000 \mathrm{MW}$, representando el $8 \%$ del suministro de energía eléctrica del país. Brasil también tiene dos plantas nucleares, que según WNA representan el 3\% de su suministro eléctrico, mientras que en México -con dos plantas- la cifra se eleva por sobre el $5 \%$. Pero, Chile tiene un problema muy específico y no menor: el pequeño tamaño de su mercado energético.

Asimismo, la mayoría de los reactores que operan en el mundo en la actualidad tienen una capacidad de por lo menos $1.000 \mathrm{MW}$, pero una regla básica expresa que para que una 
red de suministro de energía no pierda su estabilidad, ninguna planta debiera exceder de forma individual el 10\% de su capacidad total. En Chile, el Sistema Interconectado Central (SIC) que abastece a todo el país, excepto al norte y al extremo sur, actualmente tiene una capacidad bruta instalada de aproximadamente 8.000 MW.

Sin embargo la tecnología nuclear se está orientando rápidamente hacia plantas más pequeñas y ésto, en conjunto con el crecimiento de la demanda de electricidad en Chile que se está incrementando más que el PIB- haría que su utilización fuera más factible. De hecho, algunas plantas que están siendo planificadas en la actualidad son tan pequeñas como para $600 \mathrm{MW}$, un tamaño similar al de la represa hidroeléctrica de Ralco en el sur del país.

Pero la necesidad de materiales especiales y de características sofisticadas de seguridad implica que los costos de construcción de las plantas de energía nuclear tienden a ser significativamente más caros que aquellos de las plantas de combustión a carbón o a gas. Un informe publicado por la Agencia de Energía Nuclear de la OCDE y de la Agencia Internacional de Energía a principios de este año, encontró que los costos de construcción de las plantas de energía nuclear fluctúan en la actualidad desde US\$1.000/kW en la República Checa, hasta US\$ $2.500 / \mathrm{kW}$ en Japón; y promediaban US\$ $1.500 / \mathrm{kW}$. En comparación, las plantas a carbón tienen un costo entre US\$1.000 - 1.500/kW y las plantas a gas, entre US\$ $500-1.000 / \mathrm{kW}$.

En un estudio efectuado el año pasado por la Academia Real de Ingeniería del Reino Unido, se encontró que si se incluyeran los costos de compensar las emisiones de gas del efecto invernadero, la energía eléctrica generada mediante combustión de gas es más cara que la energía nuclear, y que la energía eléctrica generada mediante combustión de carbón es mucho mas costosa, aún si dicho costo no se incluye. Más aún, el estudio da cuenta de que la energía eléctrica así generada es casi un $40 \%$ más barata que la energía eólica.

Sin embargo, será muy difícil desafiar la abrumadora imagen pública negativa sobre la energía nuclear, basada en factores que van desde el desastre de Chernobyl, hasta el jefe inescrupuloso de Homero Simpson. Y más aún si se recuerdan las movilizaciones de protesta a que dio lugar el eventual transporte de plutonio por las costas de Chile. Además si se considera lo ocurrido recientemente en el caso de la planta de celulosa Arauco en Valdivia, se aprecia que los chilenos están cada vez más preocupados acerca de los posibles efectos de la industria sobre el medioambiente y conscientes del impacto que la presión pública puede tener en este campo. De modo que es muy difícil que la opinión pública pueda llegar a aceptar una planta nuclear, por lo menos en el corto y mediano plazo.

No obstante lo anterior, no es posible dejar de considerar el documentado y profundo alegato a favor de la energía nuclear que hace James Lovelock en su último libro, publicado 
a comienzos de 2006, The revenge of Gaia. ${ }^{7}$ Lovelock señala que el calentamiento global y el cambio climático son inevitables y que todas las soluciones propuestas o aplicadas hasta ahora no pueden resolver el problema (la biomasa, la energía solar, la energía eólica, etc.), lo único que nos puede dar un tiempo de respiro, aunque sólo sea para permitir la supervivencia de unos pocos, es hacer uso de la energía nuclear ahora.

«Pocos, inclusive entre los ecologistas y los climatólogos, parecen comprender la potencial gravedad de la inminente catástrofe global que se avecina. Y, entre los que lo hacen, la comprensión está sólo en la mente consciente, no genera la reacción visceral de miedo que sería de esperar.» (Lovelock 2007: 197)

\section{EI Informe Zanelli: partidarios y opositores}

La crisis energética ha logrado instalar la discusión sobre la energía nuclear en Chile en las más altas esferas políticas y científicas. Un país como Chile que aparece como un país muy vulnerable en el tema energético, requiere tener una opinión política avalada por opiniones científicas respecto del uso o no uso de la energía nuclear, o en la formulación de una política energética sustentable para el mediano plazo.

A pesar que previo a la llegada al gobierno de Chile de Michelle Bachelet, siendo ella aún candidata para ese cargo en noviembre del 2005, se reunió con ecologistas y medioambientalistas con quienes se comprometió que en su gobierno no incluiría la opción de la energía nuclear en la política energética nacional, no obstante la firma de este acuerdo, en marzo del año 2007 la ya Presidenta de Chile, convocó a un grupo de 11 científicos y expertos en diversas áreas, los cuales fueron dirigidos por el físico Jorge Zanelli, con el trabajo de recopilar información y estudiarla con el fin de dar una opinión sobre las potencialidades de la opción nuclear como parte de la solución de la crisis energética del país.

La llamada "Comisión Zanelli" entregó su informe en noviembre del 2007, en el cual se indica como principio que "el examen de todos los antecedentes de que dispuso este grupo de trabajo no permite descartar la energía nuclear como una opción energética futura para el país". ${ }^{8}$ La opción nuclear se demuestra según la experiencia internacional recogida por los expertos como segura, confiable y económicamente viable.

La entrega del "Informe Zanelli” aconsejó también la creación de una entidad asesora

\footnotetext{
${ }^{7}$ James Lovelock (2007) La Venganza de la Tierra. Por qué la Tierra está rebelándose y cómo podemos todavía salvar a la humanidad. Planeta, Santiago.

8 INFORME (2007) Grupo de Trabajo en Núcleo-Electricidad. La opción Núcleo-Eléctrica en Chile, Septiembre 2007, página 46.
} 
del Ministro de Energía en materia de energía nuclear, la cual está compuesta también por representantes de los ministerios de Defensa, Relaciones Exteriores y Medio Ambiente, además de otros expertos entre los cuales se encuentran asesores de la antigua comisión dirigida por Jorge Zanelli.

Este grupo asesor se auto-impuso una meta para el fin del mandato de Michelle Bachelet, cual es el dejar terminados los estudios en áreas sensibles como la sismología, la localización, y la aceptación pública, para que el próximo gobierno del país tenga todos los elementos y criterios para tomar una decisión al respecto de la energía nuclear en el país.

La crisis energética ha dado pie para que no sólo el sector estatal se preocupe de la energía nuclear en Chile; también desde el sector privado se han abierto una serie de intereses, sobre todo de los sectores económicos que fuertemente están invirtiendo en el sector minero del país. Estos grupos económicos se han mostrado muy proclives a invertir junto al Estado de Chile en este tipo de energía y tan sólo esperan el marco regulatorio que el Estado debiera establecer.

Por su parte el sector hidroeléctrico también ha comenzado a testear las posibilidades de la energía nuclear, sobre todo después del cúmulo de reparos que han surgido frente a las mega-centrales que Endesa y el grupo Matte tienen planificadas en la Región de Aysén en el sur de Chile.

Por otro lado están los movimientos, ONG's e instituciones ecologistas y medioambientalistas, que reafirman su negativa a esta posibilidad en vistas a los problemas ambientales, de seguridad, de desechos contaminantes y de los costos económicos que han sido detallados más arriba, y han puesto muchos reparos a los alcances de la Comisión y el Informe Zanelli.

Las dudas de los movimientos contrarios a la energía nuclear se sientan sobre las bases de un informe alternativo, liderado por Chile Sustentable, y en el cual impugnan no sólo los argumentos esgrimidos por la Comisión Zanelli, sino que en forma general, la Política Energética del país. Las objeciones de este Informe Alternativo ${ }^{9}$ se centran en las siguientes aprehensiones:

a) La generación núcleo-eléctrica dependería de un recurso no renovable como es el uranio y de los países que producen y reprocesan este combustible.

b) La generación núcleo-eléctrica no es una alternativa limpia respecto de las emisiones de $\mathrm{CO}^{2}$ (Dióxido de Carbono), ya que son significativas si se toman en

\footnotetext{
${ }^{9}$ Sara Larraín y Rosa Montero (2007) La Energía Nuclear no tiene Futuro, Fundamentos de la oposición del movimiento ambientalista a la opción núcleo-eléctrica, Chile Sustentable, Fundación Heinrich Boell. Editorial LOM, Santiago.
} 
consideración todos los ciclos del proceso de producción, desde la minería del uranio hasta la disposición final de los desechos radioactivos.

c) En las consideraciones económicas no se consideran los costos de transporte de desechos radioactivos, la disposición de esos desechos y el desmantelamiento de centrales al fin de su vida útil.

d) Que nuestro país es altamente sísmico, y supera los 7 grados Richter que establece la seguridad de la industria nuclear a nivel mundial, lo cual equivaldría a un gasto extra en infraestructura para garantizar su seguridad.

e) Por último recomiendan la adopción de una Política Energética que se oriente a la Eficiencia Energética y considere de forma más diversa la matriz energética, aumentando la consideración de las Energías Renovables No Convencionales (ERNC) dentro de ella, para evitar el casi monopolio de las centrales hidro y termo eléctricas.

\section{La integración al anillo energético latinoamericano: una opción «bolivariana»}

El gobierno de Chile ha impulsado activamente la integración de un anillo energético latinoamericano, que es un proyecto de integración energética al cual se han plegado muy rápidamente Argentina, Brasil, Uruguay y Paraguay como países demandantes y Perú como posible fuente de abastecimiento. Si bien el "anillo energético" es sólo una red de gasoductos y conexiones bilaterales entre los países que necesitan energía y aquellos a los que les sobra, y por tanto no es un real proceso de integración, comienza con ella un proceso de unificación y complementación en los temas energéticos que llevan adelante la mayoría de los países sudamericanos, especialmente en los proyectos de suministro de gas natural, y se abren así las puertas a un camino que puede fortalecer sustantivamente los procesos de integración subregional en curso. De alguna manera lo perciben así algunos gobiernos latinoamericanos, como el venezolano, que ha manifestado su interés en hacerse parte de dicho proceso.

El proyecto de "Gasoducto del Sur" previó la construcción de un gasoducto desde el sur del Perú, donde se encuentra el campo de Camisea. El proyecto requería inversiones por el orden de los 2.500 millones de dólares para construir un ducto de 1.200 kilómetros aproximadamente, desde el puerto peruano de Pisco, conectado al yacimiento gasífero Camisea, hasta el norte de Chile, el puerto de Tocopilla, donde se conectaría a redes de distribución del hidrocarburo hacia Argentina, Uruguay, Paraguay y Brasil.

Una importante parte de la apuesta regional del "anillo energético" descansaba en los estudios prospectivos realizados en la región gasífera de Camisea, donde se ubica el tercer yacimiento de gas natural del subcontinente, después de los que existen en Argentina y Bolivia. Desde esa reserva, se extrae el gas licuado de petróleo que Perú exporta hacia 
México y la costa oeste de Estados Unidos. Las reservas probadas de gas natural de la cuenca de Camisea se estima que alcanzaban en 2004 los 14.100 millones de metros cúbicos. Sin embargo, de los estudios técnicos realizados surge que el volumen de gas posible de ser explotado podría multiplicarse por dos en 2008 , fecha en la que se llegaría a los 30.000 millones de metros cúbicos.

Uno de los problemas más difíciles de resolver sería aclarar qué pasará si los clientes de un determinado país están dispuestos a pagar un mayor precio por el hidrocarburo en caso de una emergencia energética, lo que dejaría fuera del mercado a otros compradores, a lo cual se suman las dificultades de una integración política entre los países del subcontinente, otro punto que podría hacer fracasar la puesta en marcha del "anillo energético". Lo importante sin embargo es que el establecimiento de este anillo energético debería ofrecer seguridad a las empresas y a la ciudadanía. Lo que parece inaudito es que en un mundo globalizado y donde los tratados de libre comercio afloran por doquier, algunos países, entre ellos Chile, estén buscando fuentes de gas natural tan distantes como lo es el sudeste asiático, cuando Bolivia tiene recursos enterrados mucho más cercanos. Más aún si consideramos que:

«La energía ha sido el pilar básico en la construcción de la Unión Europea, ya que ha sido la primera en ignorar las fronteras. El sistema eléctrico interconectado ha sido importante no sólo en el ámbito del desarrollo económico europeo, sino que ha consolidado entre ellas un espíritu de cooperación y de compartir responsabilidades de indudable repercusión en lo social y en lo político». (Solís de Ovando y Larraín, 2005:47)

\section{El cambio hacia una matriz energética sustentable y autónoma: las energías renovables}

Desde el programa Chile Sustentable se ha venido sosteniendo, desde hace ya varios años, la necesidad de adoptar una política de fomento a las energías renovables no convencionales (ERNC), la cual tiene su justificación en la necesidad del país de reducir su vulnerabilidad y dependencia y de contar con fuentes de energía abundantes, seguras y a precios competitivos, ante la evidencia de que el mercado no ha sido suficiente para asegurar el desarrollo energético del país.

"El país requiere una política proactiva, que debe tener un doble enfoque, diversificar las fuentes de abastecimiento y optimizar el consumo. En este sentido el objetivo esencial de la ley de fomento a las ERNC, además de la preservación del medio ambiente, es disminuir la vulnerabilidad de un sector estratégico como es el sector energético del país.

La experiencia internacional indica que el estado debe jugar un rol imprescindible en el planeamiento energético de largo plazo, y además, realizar una evaluación sistemática de 
los recursos renovables, básicamente de la geotermia, la biomasa y las energías eólica y solar.

En efecto, considerando por simplicidad sólo el sector eléctrico, en el Sistema Interconectado Central (SIC) pueden desarrollarse la geotermia, la energía eólica y la biomasa. Y en el caso del Sistema Interconectado del Norte Grande (SING), pueden desarrollarse la geotermia, la energía eólica y la energía solar. El nivel de desarrollo tecnológico disponible para explotar estas fuentes de energía ya se encuentra en pleno desarrollo.

Se estima que el potencial geotérmico del país transformable en energía eléctrica excede los 8.000 MWe y que sólo en el SIC el potencial de mediano y corto plazo alcanza los 2.000 MWe.10 Una ventaja adicional de la energía geotérmica es que proporciona potencia firme, que puede ser despachada en la base del sistema eléctrico. Los riesgos y altos costos que implica la etapa de estudio y exploración inicial de la geotermia, los que pueden llegar a los 10 millones de dólares, constituyen el primer obstáculo al desarrollo de esta fuente de energía.

En el caso de la energía eólica, existe un potencial interesante en el norte del país (especialmente en el norte grande), en los valles transversales y en el sector de la VIII región al sur. El enorme desarrollo que ha tenido esta tecnología a nivel mundial ha reducido considerablemente los costos, por lo que se espera que esta fuente sea, en el corto o mediano plazo, competitiva con las fuentes de energía convencional.

En energía solar, se estima que Chile posee uno de los más altos potenciales de generación del mundo. Sin embargo, el mayor costo de esta fuente de energía avala la idea que la energía solar térmica tendrá un desarrollo posterior al de las otras ERNC.

Con respecto a los recursos en biomasa que posee el país hoy se usa en forma relevante en la industria de la celulosa para generación eléctrica en combinación y alimentación de procesos productivos que requieren calor. Sin embargo, existe una cantidad significativa de recursos que podrían ser explotados para alimentar otras empresas e incluso al servicio público, mediante el aprovechamiento de los desechos de los recursos forestales, lo que se estima que permitiría instalar unos $300 \mathrm{MWe}$.

La adopción de una ley de fomento de energías renovables se justifica no sólo por los beneficios asociados en términos de calidad, seguridad de abastecimiento, reducción de la vulnerabilidad y desarrollo tecnológico, sino también porque las tecnologías renovables pronto serán competitivas con las tecnologías convencionales si se considera, en una perspectiva de mediano plazo, los incrementos previsibles de los costos de producción y conversión de las energías convencionales, resultantes de las alzas de precios de los combustibles fósiles y las reducciones en los costos de las energías renovables" (Chile Sustentable, 2004:113-114). 
En un trabajo recientemente publicado de Pedro Maldonado et alia, ${ }^{10}$ se señala además lo siguiente; "Como se ha demostrado en el presente estudio, las ERNC constituyen una componente relevante y de alto potencial para diversificación de la matriz energética nacional, contribuyendo a la seguridad y autonomía en el abastecimiento energético. Para desarrollar y aprovechar a cabalidad estos recursos, es recomendable considerarlos como una opción estratégica, que amerita el diseño y aplicación de programas de $\mathrm{I}+\mathrm{D}+\mathrm{i}$, formación de $\mathrm{RR} / \mathrm{HH}$ y adaptaciones tecnológicas como parte de la política energética nacional.

Ello supone incentivar la capacidad científica y tecnológica del país para mejorar la transferencia y adaptación de tecnologías a través de antenas, giras tecnológicas, empoderamiento de centros tecnológicos y fortalecimiento de actores proclives al emprendimiento, que lideren las innovaciones y los quiebres tecnológicos. Ello no sólo tendrá impactos en el ámbito de la energía, sino también en actividades de generación de valor, como el turismo, la agroindustria, la industria forestal, la pesca y la minería.» (2008:65)

\section{El uso más eficiente de la energía}

Otro de los grandes objetivos nacionales que han sido planteados desde el Programa Chile Sustentable, ha sido la necesidad de avanzar hacia un uso más eficiente de la energía.

El mejoramiento de la eficiencia con que se usa la energía también debería constituir un objetivo central de las políticas energéticas de nuestro país, que presenta agudos problemas en términos de vulnerabilidad del abastecimiento, contaminación atmosférica, equidad y dependencia energética. Sin embargo los bajos costos relativos de la energía en relación al total de los costos de producción en el caso de las empresas o incluso de consumo en relación a los costos de vida de las familias no han incentivado hasta ahora la búsqueda de un uso más eficiente de la energía.

La experiencia internacional muestra la relevancia de las iniciativas gubernamentales en el tema, que incorporan instrumentos tales como agencias especializadas, normas y estándares de eficiencia, leyes y programas de eficiencia energética, acuerdos voluntarios y apoyo financiero, por nombrar algunas.

"En México, los programas de eficiencia energética desarrollados entre 1995 y 2000, permitieron alcanzar ahorros estimados en 21.900 millones de $\mathrm{kWh}$; el presupuesto para estos programas fue cercano a los 6.3 millones de dólares, mientras que los ahorros se

\footnotetext{
${ }^{10}$ Este cálculo y los siguientes ha sido realizado por el programa de Investigación de Energía (PRIEN) de la Universidad de Chile, en base a las capacitaciones tecnológicas y de recursos humanos en Chile. A la fecha, no hay un catastro oficial de los recursos energéticos renovables no convencionales.
} 
estimaron en alrededor de 360 millones de dólares. En Brasil, el programa PROCEL ha promovido la producción y el consumo eficiente de la energía eléctrica. En el período 1995-2000, el programa permitió un ahorro de $10.371 \mathrm{GWh}$, con una inversión de 318 millones de dólares, y se estima que la inversión evitada fue de 4.740 millones de dólares.

Se estima que una vez establecidas las bases para el desarrollo del uso eficiente, el potencial de ahorro en Chile, en un horizonte de 10 años, puede ser de alrededor del $6 \%$ del consumo de combustibles y del $7 \%$ del consumo eléctrico actual.

En definitiva, las sucesivas crisis energéticas en el país, los altos precios de los combustibles a nivel internacional, los problemas ambientales del uso de energías convencionales -como el cambio climático- y los instrumentos de apoyo internacional, constituyen un marco propicio para iniciar acciones que permitan reorientar el desarrollo energético nacional hacia objetivos de sustentabilidad económica, social y ambiental" (Chile Sustentable, 2004:113-114).

Una entrevista realizada a Douglas Tompkins, el millonario conservacionista, creador del Parque Pumalín en el sur de Chile, en relación a los megaproyectos hidroeléctricos que la Empresa Nacional de Electricidad S.A. (ENDESA) pretende construir en la región de Aysén, en el extremo sur de Chile, ayuda a entender las lógicas que están en juego en relación a la energía.

"Analicemos la presunción de que esta necesidad energética es una «realidad»: ¿Quién dijo que así sea y cómo lo han demostrado? Yo por lo menos estoy lejos de creerlo. Para ENDESA es conveniente presumir dicha necesidad y hacer que todos la presuman. En otros países se exigen pruebas sólidas de que se han tomado todo tipo de medidas de eficiencia energética antes de decidirse a construir nuevas fuentes de generación de energía. Es obvio, echando un mero vistazo a las políticas energéticas, que esto no se ha hecho. La escasez de energía ha sido la forma en cómo los promotores de este proyecto junto a sus aliados en el gobierno, han caracterizado este proyecto para su propio beneficio. Si uno descubre que Chile está desperdiciando energía por todas partes sin usar suficientes planes de ahorro y eficiencia energética, ni creciendo ad infinitum, te das cuenta que no hay escasez sino que incluso sobra energía. La escasez va siempre en relación a las necesidades y al consumo. Cuando no hay agua suficiente puede significar que hay una excesiva demanda, no una escasez. Si Chile Central, por ejemplo, no estuviera tan obsesionado con el crecimiento, sobre-desarrollándose a expensas de las regiones, como es el caso de estas represas en Aysén, no tendríamos esta discusión; porque Chile estaría viviendo de acuerdo a sus medios locales. Ahora el plan requiere, para este crecimiento lascivo, que se importe energía de lugares remotos del país, causando un desastre en la provincia y a lo largo de la línea de transmisión. Cuando se tiene un programa de 
desarrollo racional no se presiona más allá de lo razonable para expandirse y crecer, se encuentra el óptimo y se trata de permanecer ahí sin sobre-extenderse y sobredesarrollarse. Lo que sería prudente es una discusión sobre el sobre-desarrollo. Para los adictos al crecimiento neoliberal, la sola mención de sobre-desarrollo es una herejía, pero están llevando a todos al abismo, como es el caso ahora. Yo preferiría evitar el inevitable colapso con políticas más racionales. Este es el problema y en todas partes del mundo hemos visto a los países sobre-desarrollados yendo cada vez más lejos para cubrir sus necesidades (Irak). Si hubieran limitado su crecimiento para vivir cómodamente y sustentablemente de acuerdo a sus medios locales, no estarían gritando por agua como un pulpo gigante que repentinamente se encuentra fuera de su medio. Hay límites naturales al crecimiento, y éstos necesitan ser evaluados de antemano y considerados en los planes de crecimiento futuro. Como periodista, ¿está usted preguntándole al empresariado si es que han tomado en consideración los límites al crecimiento? Me parece a mí que la prensa está tan intoxicada con sueños de crecimiento como el sector económico y empresarial. Desde mi punto de vista hay demasiada expectación y el crecimiento se ha transformado en una droga a la cual uno se hace adicto. Chile, como cualquier otro país, necesita acotar sus expectativas de crecimiento y vivir con sus medios locales, si es que lo que se quiere es una sociedad estable en el largo plazo. Excederse, como se está haciendo, planificando estas represas en extremos del país, es peligroso y se convertirá en una bomba de tiempo para la sociedad tarde o temprano. Sería mejor parar ahora y mirar detenidamente el plan maestro energético inexistente. Es decir ¡no hay un plan maestro energético ni plan maestro país! Admitamos que no tenemos un plan y empecemos a trabajar en esto, poniendo el tema en el tapete". ${ }^{11}$

\section{El «necesario» cambio en los estilos de vida}

En diversos trabajos anteriores, tanto individuales como colectivos, hemos planteado la imprescindible necesidad de realizar un profundo cambio de las creencias en las cuales estamos anclados, un cambio de mirada y de nuestras formas de percepción de la realidad, que nos haga tomar conciencia de que nuestros actuales estilos de vida son absolutamente insustentables, en el tiempo y en términos de su universalización. El ritmo de aceleración del calentamiento global es la prueba más evidente de ello. Hace ya más de 30 años atrás un par de intelectuales suecos Backstrand e Ingestam ${ }^{12}$ se hicieron la pregunta: How much is enough?. Recientemente lo volvieron a plantear en un nuevo trabajo al cabo de 30 años, ${ }^{13}$

\footnotetext{
${ }^{11}$ Parte de la entrevista completa de La Segunda a Douglas Tompkins, la cual no fue publicada en su totalidad el Viernes 6 de enero de 2006.

${ }^{12}$ Bäckstrand, G. e Ingelstam, L., «How much is enough? - another Sweden» en Development Dialogue 1975, $\mathrm{N}^{\circ} 1 / 2$, Dag Hammarskjöld Foundation, Uppsala.

${ }^{13}$ Göran Bäckstrand y Lars Ingelstam (2006), «Enough! Global challenges and responsible lifestyles» en What Next. Setting the context. Volume I. Development Dialogue, $\mathrm{N}^{\circ} 47$, june 2006, Dag Hammarskjöld Foundation, Uppsala. 
«...nuestros estilos de vida materialistas no contribuyen ni a la salud ni a la felicidad. En un concreto, sentido mensurable, podríamos estar mejor si reorganizáramos nuestras prioridades lejos del «crecimiento» y la expansión material. Es algo preocupante que los reparos a este efecto son aún a menudo vistos como marginales, y calificados en el debate con epítetos tales como irrealista, utópico o mirando al pasado. Ya que el sentido común y la evidencia científica en los hechos apuntan en la dirección opuesta, nosotros no vacilamos en nada para terminar como partimos. ¡Suficiente es suficiente! (2006:147)

Adela Cortina ha planteado algo similar en su libro Por una ética del consumo, señalando que es necesario que ella deba estar anclada en tres escalones básicos:

«la igualdad de consumo, entendida como la creación de estilos de vida incluyentes y universalizables; la moderación de consumo compulsivo, y el diseño de un pacto global sobre el consumo que haga posible promover la capacidad de las personas de consumir de forma autónoma, defender sus intereses mediante el diálogo y desarrollar sus proyectos de vida feliz.» (2002:304)

La propuesta hecha desde el Programa Conosur Sustentable sobre la línea de dignidad $^{14}$ apunta en la misma perspectiva: construir un horizonte ético político que limite el sobreconsumo de los países y personas ricas y así haga posible reducir el subconsumo de los pobres. Sería algo absolutamente digno de asombro, para un observador extraterrestre, comprobar nuestra insensibilidad moral como sociedades y ciudadanos frente al derroche y despilfarro de los grupos dominantes en las sociedades actuales. Qué decir de las guerras y la violencia desatada sobre poblaciones civiles debido a la disputa por el control del petróleo, como lo es la actual guerra de Irak; o por la hegemonía política del bloque occidental, como ha ocurrido en nuestro continente en el pasado reciente.

Cada día se hace más evidente la señera intuición de Gandhi respecto a que tenemos suficientes recursos para atender las necesidades de todos, pero no para dar cuenta de la codicia de unos pocos. El salto moral que requerimos como especie, como sociedad y como ciudadanos del mundo, es transitar desde la prudencia a la cordura, para tomar conciencia de que estamos enfermos de sobredesarrollo, como lo señala Lovelock:

«El crecimiento económico crea la misma adicción entre los políticos que la heroína en los toxicómanos... economistas y políticos deben conciliar la perentoria necesidad de un cese rápido y controlado de las emisiones producidas por el consumo de combustibles fósiles con las necesidades de la civilización humana.» (2007:216)

\footnotetext{
${ }^{14}$ VV.AA. (2003) Línea de Dignidad. Desafíos Sociales para la Sustentabilidad, Conosur Sustentable, Santiago. 
Sostiene que para lograr lo anterior debería recurrirse a algún sustituto adecuado, una especie de metadona económica, y señala que internet, los teléfonos móviles y los entretenimientos mediante ordenadores están cumpliendo ese papel. Hay también sugeridos otros caminos en trabajos de Rifkin, Harman y García Roca. De este último recuperamos su propuesta de abajamiento, que significa la disposición de todos quienes estamos situados en niveles de consumo no universalizables, y en consecuencia, no democráticos, para reducir nuestro consumo a fin de hacer viable de ese modo la vida de todos los seres humanos no incluidos, nuestros coetáneos y también -no lo olvidemos- nuestros descendientes.

Mirado entonces el problema debatido en este trabajo, desde la perspectiva sugerida en este último acápite, el rol que puede jugar Chile en las dinámicas de integración subregional es fundamental. Actuar como sociedad, por ejemplo, con generosidad frente a la demanda de acceso al mar de Bolivia, haría posible desatar sinergias integrativas inimaginables, y encontrar soluciones realistas e inmediatas a nuestros propios problemas energéticos. 


\section{Bibliografía}

Azzopardi, Tom (2005) «Energía Nuclear: ¿Es Viable en Chile?» en Business Chile, №230, Diciembre 2005, ver en http://www.businesschile.cl/portada.php?w=old\&id=188\&lan=es

Bitar, Sergio (1988) Chile para todos, Editorial Planeta, Santiago.

Campero, Guillermo (1978) Entre la sobrevivencia y la acción política, Editorial ILET, Santiago, págs. 35-36.

Castells, Manuel (2005) Globalización, desarrollo y democracia: Chile en el contexto mundial, FCE, Santiago.

Claude, Marcel (1997) Una vez más la Miseria. ¿Es Chile es un país sustentable?, LOM Ediciones, Santiago.

Cortina, Adela (2002), Por una ética del consumo, Taurus, Madrid.

Díaz, Marcelo (2005) "Desafíos para la política exterior de Chile en el año 2005” en Diplomacia, N 102, AbrilJunio 2005, Academia Diplomática de Chile, Santiago.

El Mercurio (2006) “¿El nuevo sueldo de Chile? en Revista del Campo, Año XXX, № 1539, 9 de enero de 2006.

García Roca, Joaquín (1998) Exclusión social y contracultura de la solidaridad. Ediciones HOAC, Madrid.

Harman, Willis (1993) «Doing Business in a Transforming Society» publicado en ICIS FORUM, Volume 23, Number 1, Winter 1993.

Lovelock, James (2007) La Venganza de la Tierra. Por qué la Tierra está rebelándose y cómo podemos todavía salvar a la humanidad. Planeta, Santiago.

Pinto, Aníbal (1959) Chile, un caso de desarrollo frustrado, Editorial Universitaria, Santiago.

Programa Chile Sustentable (2004), Situación de la energía en Chile: desafíos para la sustentabilidad, Santiago.

Quiroga, Rayén (1994) El Tigre sin Selva: consecuencias ambientales de la transformación económica de Chile: 1974-1993, Instituto de Ecología, Santiago.

Quiroga, Rayen y Van Haurwermeiren, Saar (1996) Globalización e Insustentabilidad. Una mirada desde la economía ecológica, Instituto de Ecología Política, Santiago.

Rifkin, Jeremy (1990) Entropía. Hacia el mundo invernadero, Urano, Barcelona

Rodríguez, Isabel (2005) “Apec 2004. Desde la regionalización al regionalismo" en Diplomacia, № 102, AbrilJunio 2005, Academia Diplomática de Chile, Santiago.

Rodríguez, Isabel y Pressaco, Carlos (2004) “Análisis de la Situación de Chile en los Procesos de Integración Regional: su asociación con el MERCOSUR en Informe sobre el Estado de la integración 2003 Situación de los Procesos de Integración en América Latina: Avances y Desafios, Instituto Internacional de Integración, Bolivia, 2004

Solís de Ovando, Lino y Larraín, Sara (2005) Anillo Energético Sudamericano. Desafíos para la integración energética del Cono Sur. Programa Chile Sustentable, Heinrich Boell Foundation y Conosur Sustentable, Santiago.

Tironi, Eugenio (1990) “Crisis, Desintegración y Modernización” en Proposiciones No 18, Chile, Sociedad y Transición, Ediciones SUR, Santiago. 
REVISTA POLIS

VOL. 7 No 21,2008

PRÒLOGO, págs., 37-62

( ) EDITORIAL DE LA UNIVERSIDAD BOLIVARIANA DE CHILE, 2008

VV.AA. Línea de Dignidad. Desafíos Sociales para la Sustentabilidad (2003), Conosur Sustentable, Santiago

INFORME (2007), Grupo de Trabajo en Núcleo-Electricidad. La opción Núcleo-Eléctrica en Chile, Septiembre 2007. Versión PDF en http://www.cne.cl/noticias/otros/Informe.pdf

Sara Larraín y Rosa Montero (2007) La Energía Nuclear no tiene Futuro, Fundamentos de la oposición del movimiento ambientalista a la opción núcleo-eléctrica, Chile Sustentable, Fundación Heinrich Boell. Editorial LOM, Santiago. Versión en PDF disponible en:

http://www.chilesustentable.net/sitio/pics/publicaciones/718/libro_nuclear.pdf 